\title{
Discharge Profiles of Ventral Tegmental Area GABA Neurons during Movement, Anesthesia, and the Sleep-Wake Cycle
}

\author{
Rong-Sheng Lee, ${ }^{1}$ Scott C. Steffensen, ${ }^{1,2}$ and Steven J. Henriksen ${ }^{1}$ \\ ${ }^{1}$ Department of Neuropharmacology, The Scripps Research Institute, La Jolla, California 92037, and 2Department of \\ Psychology and The Neuroscience Center, Brigham Young University, Provo, Utah 84602
}

\begin{abstract}
Although mesolimbic dopamine (DA) transmission has been implicated in behavioral and cortical arousal, DA neurons in the ventral tegmental area (VTA) and substantia nigra pars compacta (SNc) are not significantly modulated by anesthetics or the sleep-wake cycle. However, VTA and SN non-DA neurons evince increased firing rates during active wakefulness (AW) and rapid eye movement (REM) sleep, relative to quiet wakefulness. Here we describe the effects of movement, select anesthetics, and the sleep-wake cycle on the activity of a homogeneous population of VTA GABA-containing neurons during normal sleep and after $24 \mathrm{hr}$ sleep deprivation. In freely behaving rats, VTA GABA neurons were relatively fast firing (29 $\pm 6 \mathrm{~Hz}$ during AW), nonbursting neurons that exhibited markedly increased activity during the onset of discrete movements. Adequate anesthesia produced by administration of chloral hydrate, ketamine, or halothane significantly reduced
\end{abstract}

The ventral tegmental area (VTA) is the source of dopamine (DA)-containing neurons that project to structures in the ventral striatum, hypothalamus, and prefrontal association cortex, known collectively as the mesocorticolimbic DA system. This neural circuit has been implicated in mediating several motivated behaviors (for review, see Mogenson, 1987; Wise and Rompre, 1989). In this context, midbrain DA neurons in the VTA and substantia nigra pars compacta ( $\mathrm{SNc}$ ) respond to alerting, activating, and reward-related stimuli (Trulson and Preussler, 1984; Schultz, 1986; Freeman and Bunney, 1987; Schultz et al., 1993). Although mesolimbic DA transmission has been implicated in behavioral (for review, see Kalivas et al., 1993) and electrocortical (Radulovacki et al., 1979; Kropf et al., 1989; Kropf and Kuschinsky, 1991; Sebban et al., 1999a,b) activation, the firing rate of DA neurons in the VTA and SNc is not significantly modulated by the sleepwake cycle or anesthetics (Miller et al., 1983; Steinfels et al., 1983). However, VTA and SNc non-DA neurons evince increased firing rates during active wakefulness (AW) and rapid eye movement (REM) sleep, relative to quiet wakefulness (QW) (Miller et al., 1983).

Although some progress has been made in elucidating the role of DA neurons in arousal and reinforcement, relatively less is

\footnotetext{
Received Sept. 1, 2000; revised Dec. 11, 2000; accepted Dec. 19, 2000.

This work was supported by National Institutes of Health Grants DA08301 and AA06420 to S.J.H. and AA10075 to S.C.S. We thank Dr. Salvador Huitron for critical discussions of the data, and Pete Griffin and Sarah Stobbs for help with histology and the sleep deprivation experiments.

Correspondence should be addressed to Dr. Steven J. Henriksen, Department of Neuropharmacology (CVN-13), The Scripps Research Institute, 10550 North Torrey Pines Road, La Jolla, CA 92037. E-mail: steven@scripps.edu.

Copyright (C) 2001 Society for Neuroscience $0270-6474 / 01 / 211757-10 \$ 15.00 / 0$
}

VTA GABA neuron firing rate and converted their activity into phasic 0.5-2.0 sec ON/OFF periods. VTA GABA neuron firing rate decreased $53 \%$ during slow-wave sleep (SWS) and increased $79 \%$ during REM, relative to AW; however, the discharging was not synchronous with electrocortical $\alpha$ wave activity during AW, $\delta$ wave activity during SWS, or $\gamma$ wave activity during REM. During deprived SWS, there was a direct correlation between increased VTA GABA neuron slowing and increased $\delta$ wave power. These findings indicate that the discharging of VTA GABA neurons correlates with psychomotor behavior and that these neurons may be an integral part of the extrathalamic cortical activating system.

Key words: ventral tegmental area; anesthesia; slow-wave sleep; rapid eye movement sleep; sleep deprivation; GABA; cortical activation known regarding the role of midbrain non-DA neurons in these behaviors. Midbrain neurons that are negative for tyrosine hydroxylase staining lie in close proximity to tyrosine hydroxylasepositive DA neurons. It has been suggested that these non-DA neurons are GABAergic neurons (Nagai et al., 1983; Otterson and Storm-Mathisen, 1984; Mugnaini and Oertel, 1985). GABAmediated responses have been implicated in the modulation of the sleep-wake cycle (Nishikawa and Scatton, 1985). Increases in GABA release during slow-wave sleep (SWS) have been observed in the posterior hypothalamus (Nitz and Siegel, 1997), an area implicated in the regulation of behavioral arousal (Szymusiak and McGinty, 1986). Microinjection of GABA agonists into the posterior hypothalamus produce hypersomnia in the cat (Lin et al., 1989). Significantly, GABAergic neurons projecting to the posterior hypothalamus arise in the VTA and SNc (Ford et al., 1995). GABAergic neurons likely play a critical role in the modulation of DA mesocorticolimbic neurotransmission, which has recently been implicated in the control of REM sleep in the canine model of narcolepsy-cataplexy (Nishino and Mignot, 1997).

We have recently characterized, in anesthetized rats, a homogeneous population of VTA non-DA neurons that contain GABA, connect to DA neurons, and project to corticolimbic structures (Steffensen et al., 1997, 1998). They were distinguished electrophysiologically from DA neurons by their rapid-firing, nonbursting activity, short-duration action potentials, EPSPdependent spontaneous spikes, and lack of spike accommodation to depolarizing current pulses. To evaluate the potential role of VTA GABA neurons in cortical arousal and psychomotor behavior, we studied the discharge profiles of these neurons during the induction and maintenance of adequate anesthesia, during elec- 
trocortical rhythmic activity, and during the sleep-wake cycle in normal and sleep-deprived unrestrained rats.

Preliminary results have been published previously (Steffensen et al., 1996; Lee et al., 1997).

\section{MATERIALS AND METHODS}

Animal care. Nineteen male Sprague Dawley rats (Charles River Laboratory, Hollister, CA) weighing 300-500 gm were housed individually with ad libitum access to food and water and were maintained on a reverse $12 \mathrm{hr}$ light/dark cycle (off at 10:00 A.M., on at 10 P.M.). Animal care, maintenance, and experimental procedures were in accordance with the Scripps Research Institute Animal Research Committee (IACUC approved; Animal Welfare Assurance no. A3194-01).

Microwire electrode implantation surgery and single-unit recording. Rats were anesthetized with sodium pentobarbital $(50 \mathrm{mg} / \mathrm{kg}$, i.p.) for microwire implantation surgery. Eight stainless steel Teflon-insulated microwires $(50-62 \mu \mathrm{m})$ assembled in a single bundle (diameter of splayed microwires tip is $0.75 \mathrm{~mm}$; NB Labs, Denison, TX) were connected to a pin on one or two strip connectors. Microwire bundles were lowered into the VTA [ -5.6 to $-6.2 \mathrm{~mm}$ anteroposterior, $0.7-1.0 \mathrm{~mm}$ mediolateral, and $7.8 \mathrm{~mm}$ from the cortical surface (Paxinos and Watson, 1986)] (Nagai et al., 1983; Otterson and Storm-Mathisen, 1984; Mugnaini and Oertel, 1985). EEG leads $(120 \mu \mathrm{m})$ were connected to screws implanted in the cranium over right retrosplenial and frontal cortices and left parietal and frontal cortices. EMG wires $(120 \mu \mathrm{m})$ were threaded $1-2 \mathrm{~cm}$ into the neck muscles. Rats were given at least 1 week to recover after surgical implantation and to habituate to daily handling.

Single-unit, EEG, and EMG recordings. Spontaneous single VTA neuron spikes were recorded from unrestrained rats using a detachable headset containing unity-gain field effect transistors, one for each of the 16 microwire electrodes. Action potential signals obtained from VTA neurons were propagated through a 25 -channel commutator, filtered at $1-3 \mathrm{kHz}(-3 \mathrm{~dB})$ by an Axon Instruments (Foster City, CA) CyberAmp 380 amplifier, isolated by a window discriminator (River Point Electronics, Dudley, NC), digitized by National Instruments NB-MIO-16 and PCI-MIO-16 multifunction data acquisition boards at $20 \mathrm{kHz}(12-$ and 16-bit resolution), and processed on- and off-line by customized National Instruments (Austin, Texas) LabVIEW virtual instrument spike detection software installed in MacIntosh and Pentium III computers. EEG (filtered at $0.3-100 \mathrm{~Hz}$ at $-3 \mathrm{~dB}$ ), EMG (filtered at $5-35 \mathrm{~Hz}$ at $-3 \mathrm{~dB}$ ), and piezoelectric activity $(0.1-100 \mathrm{~Hz}$; transducer cemented to underside of the suspended floor of the chamber) were recorded differentially and amplified 100-10000 times by an Axon Instruments CyberAmp 380 Amplifier. Responses were subsequently displayed on Tektronix digital/ analog storage oscilloscopes and a Grass Model 8-16 polygraph, digitized at $200 \mathrm{~Hz}$ (12- and 16-bit resolution) on National Instruments NB-MIO-16 and PCI-MIO-16 multifunction data acquisition boards, and processed on- and off-line by customized National Instruments LabVIEW EEG analysis software installed on MacIntosh and Pentium III computers. The duration of the recording sessions was 2-3 hr. A video recording system consisting of a camcorder (Sony CCD-TR7), videographics cards (Mass Microsystems Colorspace II/FX), a MacIntosh Quadra 950 computer, a video monitor, and a videocassette recorder was used to monitor rat behavior. Graphical windows displaying spike rate meter, spike interval, and EEG spectrograms were superimposed on the video signal for off-line correlation of behavior with electrophysiological responses.

Data analysis and statistics. Data presented in this report were obtained from 19 unrestrained rats. Spike activity, EEG, EMG, and piezoelectric activity recorded by computer were displayed and analyzed by IGOR Pro software (Wavemetrics, Lake Oswego, OR). EEG activity was recorded from electrodes located over the retrosplenial, parietal, and frontal cortices; however, only retrosplenial to contralateral frontal recordings were subjected to analysis. EEG voltage and frequency spectra were generated from $4 \mathrm{sec}$ activity epochs by fast root-mean-square (rms) and Fourier transform processing algorithms. Frequency spectral bands were extracted from the Fourier analysis at $1-4 \mathrm{~Hz}(\delta), 4-8 \mathrm{~Hz}(\theta), 8-18 \mathrm{~Hz}$ $(\alpha)$ and $30-58 \mathrm{~Hz}(\gamma)$ for every 4 sec epoch. For comparisons between normal and deprived sleep, we averaged the $\delta, \alpha$, and $\gamma$ activity of all 4 sec EEG epochs during 2 min of SWS, AW, and REM sleep. $\delta, \alpha$, and $\gamma$ activity were determined only during SWS, AW, and REM sleep, respectively.

Single-unit firing rate was calculated as the average spikes per second over 2 min of recording. Two minutes was chosen to normalize to the short duration of REM episodes relative to AW and SWS. The calculation of the predominant instantaneous firing frequency was determined from the first-order interspike interval histograms as well as by integration of rate meter records. Control and anesthetic effects were measured immediately before handling of the animals for administration of anesthetic and 5 min after adequate anesthesia, as determined by the lack of response to brisk tail pinch. Assessment of rhythmic and higher-order interspike interval tendencies was performed with autocorrelation histograms and with first-order interspike interval histograms, on the same data segments as for the other unit calculations. Classification of phasic activity during anesthesia was accomplished by consulting the raw records, together with the instantaneous ( $0.1 \mathrm{sec}$ rate sampling) rate meter records and first-order interspike interval histograms to characterize the predominant firing pattern. Spike-triggered averaging (STA) was used to estimate the extent of cross-correlation between spikes and EEG activity. The time of each individual spike was used as a reference to gather and average concomitant windows of EEG data (usually $1.5 \mathrm{sec}$ before and after the spike; normalized to 100 spike events across state), thus allowing estimation of the EEG pattern, which is associated preferentially with any given spike discharge. The results were compared before and after drug treatment using two-way ANOVA, without replication $(\alpha=0.05)$. Figures were compiled with Igor Pro software.

Sleep deprivation procedure. Electroencephalographic and single-unit activity were recorded simultaneously in 6 of the 19 rats during a sleep-wake cycle before and after $24 \mathrm{hr}$ of sleep deprivation. The last sleep-wake cycle before sleep deprivation was recorded between 8:00 A.M. and 12 P.M. during the reverse $12 \mathrm{hr}$ light/dark cycle (off at 10:00 A.M., on at 10:00 P.M.). After an episode of normal REM sleep, each rat was awakened and housed together with the other rats in a $3 \times 3 \times 2$ foot, open-field box. Their activity was monitored continuously. They were constantly handled and exposed to novel objects and alerting stimuli during the $24 \mathrm{hr}$ period of sleep deprivation. Every $2-3 \mathrm{hr}$, each rat was connected to the recording apparatus, and EEG and single-unit activity were monitored but no SWS was allowed. After a minimum of $24 \mathrm{hr}$ of sleep deprivation, each rat was again connected to the recording apparatus and allowed to sleep. Care was taken to record the deprivation sleep between 8:00 A.M. and 12 P.M..

Histology. At the termination of the chronic recordings, electrolytic lesions ( $\pm 3 \mathrm{~mA} ; 10-15 \mathrm{sec}$; Stimulator S88 and Isolator Unit PSIU 6, Grass Instrument, Quincy, MA) were passed through the recording electrode during deep anesthesia to verify its location in the VTA region. The animals were subsequently administered a lethal dose of halothane anesthesia or pentobarbital, and the brains were removed and preserved in $10 \%$ formalin. The brains were frozen and sectioned in a cryostat into $50 \mu \mathrm{m}$ slices for inspection of the lesion site.

\section{RESULTS

Extracellular electrophysiological characterization of
VTA GABA neurons

We have previously described the electrophysiological, neurochemical, and ultrastructural characteristics of VTA GABA neurons in anesthetized (Steffensen et al., 1998) and freely behaving rats (Gallegos et al., 1999). In brief, VTA GABA neurons recorded in halothane-anesthetized rats represent a homogeneous population of phasic (only when anesthetized; see below), rapidfiring, nonbursting, short duration $(<500 \mu \mathrm{sec})$ action potential neurons that connect to VTA DA neurons and receive excitatory input from the cortex and hippocampus. The most distinguishing feature of VTA GABA neurons recorded in halothaneanesthetized rats was their uninterrupted phasic activity characterized by alternating $0.5-2.0 \mathrm{sec}$ ON/OFF periods (Fig. $1 B$ ) (Steffensen et al., 1998). In freely behaving rats, VTA GABA neurons do not exhibit phasic activity (Fig. 1C) (Gallegos et al., 1999). They can be classified as VTA GABA neurons based on their spiking characteristics and by response to afferent input. As in anesthetized rats, VTA GABA neurons are relatively rapidfiring neurons. The range of firing rates of all VTA GABA neurons recorded in this study during AW ranged from 4 to 65 


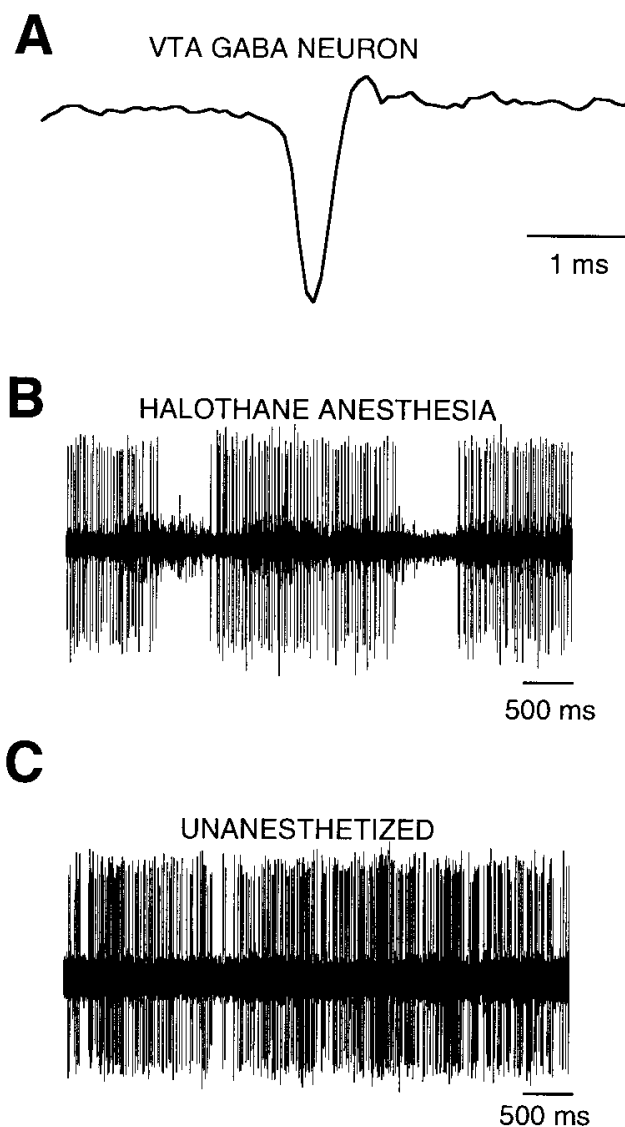

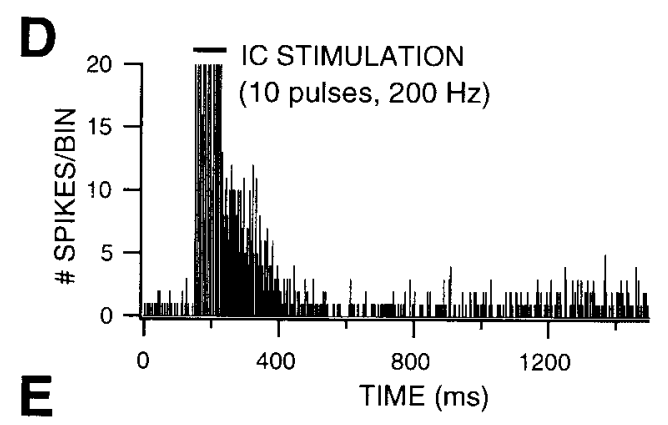
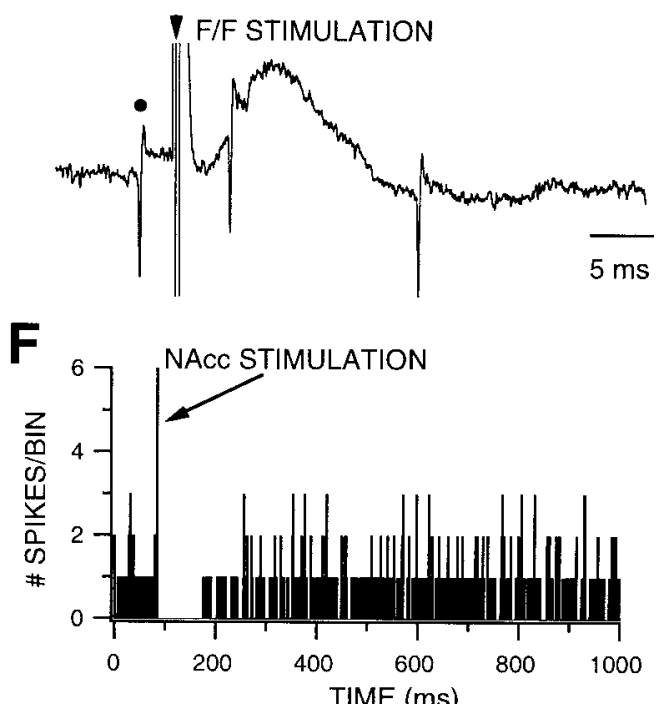

Figure 1. Characterization of VTA GABA neurons in freely behaving rats. $A$, This trace shows a representative unfiltered recording of a VTA GABA neuron spike obtained in a freely behaving rat. Spikes were of short duration $(<500$ $\mu \mathrm{sec})$ with an initial large negative deflection followed by a smaller positive potential. $B$, This trace shows a 5.0 sec filtered recording of a VTA GABA neuron recorded in a halothane-anesthetized rat restrained in a stereotaxic apparatus. Note the phasic firing characteristic of all VTA GABA neurons recorded in anesthetized rats (Steffensen et al., 1998). The phasic firing was characterized by $0.5-2.0 \mathrm{sec}$ ON and OFF periods that persisted through the recording session. $C$, This trace shows a $5.0 \mathrm{sec}$ filtered recording of a VTA GABA neuron recorded in an unrestrained, unanesthetized rat. Note that the firing rate is regular and nonphasic. $D$, This peristimulus histogram shows the activity of a VTA GABA neuron over 10 cumulated epochs of high-frequency stimulation of the internal capsule (IC STIMULA$T I O N ; 10$ pulses at $200 \mathrm{~Hz}$ ). Although obscured by the artifact at this scale, this neuron was driven orthodromically by each stimulus pulse at a latency of 2-3 msec. However, what is more obvious is the occurrence of multiple spike discharges after the stimulus train, the number of which was manifold greater than the number of stimulus pulses within the

train. Stimulation intensity was $2 \times$ the threshold for orthodromic activation. $E$, This trace shows a representative unfiltered recording of a VTA GABA neuron spontaneous spike ( marks spontaneous spike) and fimbria/fornix-elicited (F/F STIMULATION) spikes (arrowhead marks f/f stimulus artifact). All $\mathrm{f} / \mathrm{f}$-driven VTA GABA neuron spikes were orthodromic as demonstrated in this example, wherein the spontaneous spike fails to extinguish either of the f/f-driven spikes. A small field potential was also elicited in the VTA after f/f stimulation. Short-latency VTA GABA neuron spikes occurred on the initial rising edge of the field potential, whereas long-latency VTA GABA neuron spikes occurred in the small negative depression after the initial peak. $F$, This peri-event histogram shows the activity of a VTA GABA neuron over 10 cumulated epochs of single stimulation of the NAcc. Stimulation of the NAcc rarely evoked VTA GABA neuron spikes but produced a distinct period of inhibition of their spontaneous activity.

$\mathrm{Hz}$, with a mean of $28.7 \pm 5.6 \mathrm{~Hz}(n=25)$. This was significantly higher $(p<0.05)$ than the mean firing rate of $19 \mathrm{~Hz}$ reported previously for VTA GABA neurons recorded in halothaneanesthetized rats (Steffensen et al., 1998). Similar to VTA GABA neurons recorded in anesthetized rats, VTA GABA neurons were characterized by nonbursting, short-duration $(<500 \mu \mathrm{sec})$ spikes (Fig. 1A). Spike characteristics, as well as anatomical localization to the VTA, were the primary criteria used to classify the neurons as VTA GABA neurons. In addition to the primary spiking criteria (i.e., initial negative-going spike waveforms, $<500$ $\mu$ sec spike duration, nonbursting, relatively fast firing), we also established secondary criteria based on their response to afferent input. VTA GABA neurons were identified as such by at least one of the following stimulation criteria: multiple spiking after high-frequency stimulation of the internal capsule (IC); duallatency spiking after single stimulation of the fimbria/fornix (f/f); or inhibition of spontaneous activity by single stimulation of the nucleus accumbens (NAcc). VTA GABA neurons were consistently driven orthodromically or antidromically, or both, by single stimulation of the IC. Short trains of high-frequency IC stimulation $(10$ pulses at $200 \mathrm{~Hz})$ elicited multiple spike discharges that occurred with latencies nearly an order of magnitude greater than their single-spike antidromic or orthodromic latency of 2-3 msec (Fig. 1D). We have previously demonstrated that IC-stimulated multiple spiking is blocked by systemic MK-801 or in situ micro- electrophoretic application of APV, indicating that the ICstimulated input is mediated by NMDA receptors (Steffensen et al., 1998). VTA GABA neuron spikes are also elicited orthodromically by fimbria/fornix stimulation at dual latencies (mean latency $=6.2 \pm 1.1 \mathrm{msec}$ and $22 \pm 2.3 \mathrm{msec} ; n=7$ ) (Fig. $1 E)$. Finally, these neurons could also be identified in the freely behaving rat by the inhibition of their spontaneous activity after stimulation of the NAcc (Fig. $1 F$ ) (mean duration of inhibition = $82 \pm 7 \mathrm{msec} ; n=6$ ). All neurons classified as VTA GABA neurons met the criteria for spike characteristics and either were driven by IC or $\mathrm{f} / \mathrm{f}$ stimulation or inhibited by NAcc stimulation.

\section{Effects of movement and anesthetics on VTA GABA neuron spontaneous activity}

We observed that the firing rate of VTA GABA neurons was phasically modulated by diverse forms of motor activity. The type of movement was not quantitatively examined; however, marked accelerations in firing rate were associated with the onset of certain movements such as head orienting or forelimb movement or transitions from SWS to AW. On the other hand, little variation in firing rate was observed with transitions to or during sustained locomotor activity. During phasic motor activity the firing rate of each neuron increased dramatically. The rate meter in Figure $2 A$ depicts the firing rate of three VTA GABA neurons recorded 
Figure 2. Effects of movement and anesthesia on VTA GABA neurons. $A$, Insets $i$ and $i i$ show $5.0 \mathrm{sec}$ "filtered" recordings of the spontaneous activity of the two most rapidly firing VTA GABA neurons shown on the rate meter record, recorded from the same electrode, before and after chloral hydrate anesthesia. The traces were taken during the time marked on the rate meter record. Note the slowing of firing rate and the phasic activity during adequate anesthesia. The rate meter record demonstrates the firing rate of three VTA GABA neurons recorded simultaneously from microwire electrodes chronically implanted in a freely behaving rat during movement and the induction of anesthesia produced by chloral hydrate. Although the firing rates of the three VTA GABA neurons differed, all were characterized by marked increases in firing during movement (horizontal bars $)$. Chloral hydrate $(200 \mathrm{mg} / \mathrm{kg})$ suppressed the firing of two VTA GABA neurons and modestly decreased the firing of the remaining neuron in this rat. Adequate anesthesia was determined by the lack of reflex response to tail pinch. $B$, Compared with saline, adequate anesthesia produced by intraperitoneal chloral hydrate or ketamine $(150 \mathrm{mg}$ / $\mathrm{kg}$ ) and exposure to halothane vapor $(1 \%$ in $4 \times 4 \times 10$ inch Plexiglas box $)$ significantly decreased VTA GABA neuron firing rate by 86,62 , and $45 \%$, respectively. Asterisks indicate significance level $p<0.05$. $C, D$, These rate
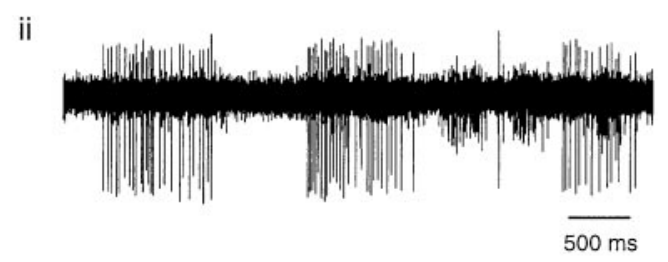

A
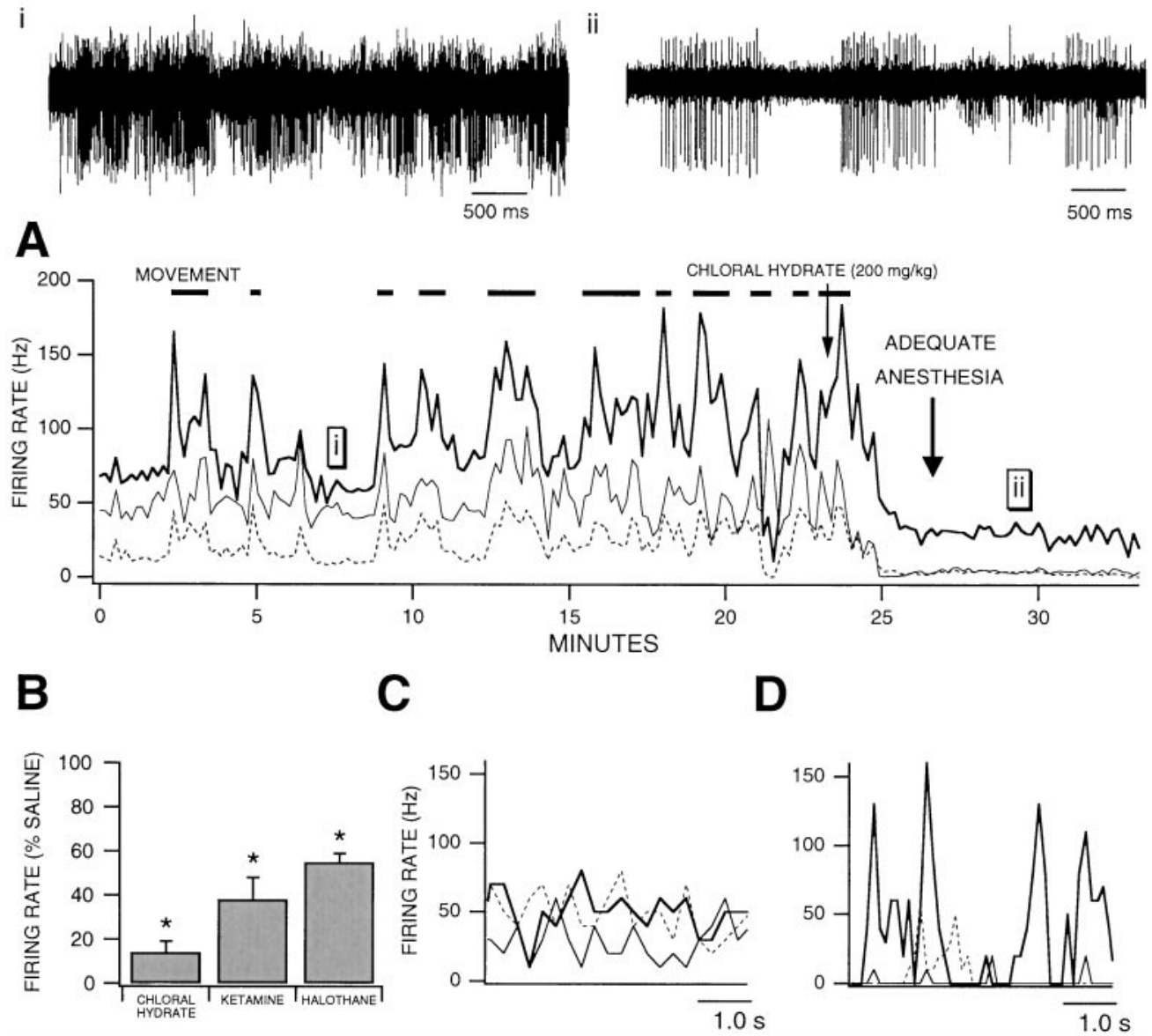

D

$1.0 \mathrm{~s}$

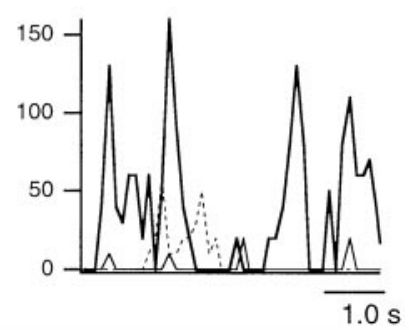

meter records show $5.0 \mathrm{sec}$ epochs of the instantaneous firing rates of the same three simultaneously recorded VTA GABA neurons before $(C)$ and after $(D)$ the induction of chloral hydrate anesthesia. Note that before anesthesia the firing rate of each spike is relatively regular even when sampled at 100 msec time bins. After adequate anesthesia, the regular firing rate is transformed into pronounced phasic (ON/OFF) activity. Indeed, in one cell there are paroxysms of increased firing during the ON periods of phasic activity; however, when averaged over 2 min there is a definite slowing of firing rate, likely caused by the suppression of firing during the OFF period of phasic activity. Often, the ON period of phasic firing during anesthesia is characterized by an initial acceleration of firing that likely gives rise to the transients, followed by an adaptation and then an abrupt OFF period.

simultaneously in the same rat during AW/QW and during the induction of anesthesia by chloral hydrate. During movement the firing rate of each neuron increased dramatically. The mean increase was $85 \pm 6 \%(n=14)$. Spontaneous firing rates often eclipsed $100 \mathrm{~Hz}$ for $10-20 \mathrm{sec}$. Systemic administration of 200 $\mathrm{mg} / \mathrm{kg}$ chloral hydrate markedly decreased the firing rates of these three neurons during adequate anesthesia, as determined by the absence of reflex activity associated with a brisk tail pinch. Figure $2 B$ summarizes the effects of chloral hydrate, ketamine, and halothane anesthesia on the firing rate of VTA GABA neurons. Compared with a period of QW immediately before handling of the animals for administration of anesthesia, all three anesthetics significantly reduced the firing rate of VTA GABA neurons as follows: chloral hydrate, $86 \%\left(p=0.005 ; F_{(2,11)}=\right.$ $22.806)$; ketamine, $62 \%\left(p=0.013 ; F_{(2,11)}=14.25\right)$; and halothane, $45 \%\left(p=0.047 ; F_{(2,11)}=6.835\right)$. Anesthesia produced by chloral hydrate, ketamine, and halothane also produced phasic ON/OFF activity as shown in the representative recordings of a VTA GABA neuron before and after chloral hydrate anesthesia. This is also shown in the rate meter records in Figure 2, $C$ and $D$, which depict the instantaneous (100 msec time bins) firing rate of the three simultaneously recorded VTA GABA neurons before and after chloral hydrate.

\section{VTA GABA neuronal activity during the sleep-wake cycle}

Active wakefulness was recognized by low-voltage, desynchronized EEG activity, increased EMG activity, locomotor activity, upright posture, open eyes, and responsiveness to sound or touch (Fig. 3). SWS was characterized by the presence of high-voltage, synchronized EEG activity, recumbent posture, closed eyes, and diminished EMG activity. Rapid eye movement sleep was characterized by low-voltage desynchronized EEG, continued behavioral signs of sleep, and a decrease in EMG activity to the level of background noise. The discriminated unit activity of a relatively slow VTA GABA neuron recorded simultaneously with the EEG and EMG activity is also shown. The discharging of this VTA GABA neuron is modulated by the stage of sleep. Figure 4 shows the firing rate of a more typical, rapidly firing VTA GABA neuron during multiple sleep-wake cycles over $>3 \mathrm{hr}$. The firing rate was modulated by movement during $\mathrm{AW}$, was regular during SWS, and was consistently elevated during REM episodes. Figure $5 A$ shows the firing rates of all 25 VTA GABA neurons studied during the sleep-wake cycle. To summarize, VTA GABA neuron firing rate decreased significantly $\left(p=0.0012 ; F_{(2,49)}=13.475\right)$ during SWS and increased significantly $\left(p=0.042 ; F_{(2,49)}=\right.$ 4.602) during REM sleep, relative to AW (Fig. $5 B$ ). 

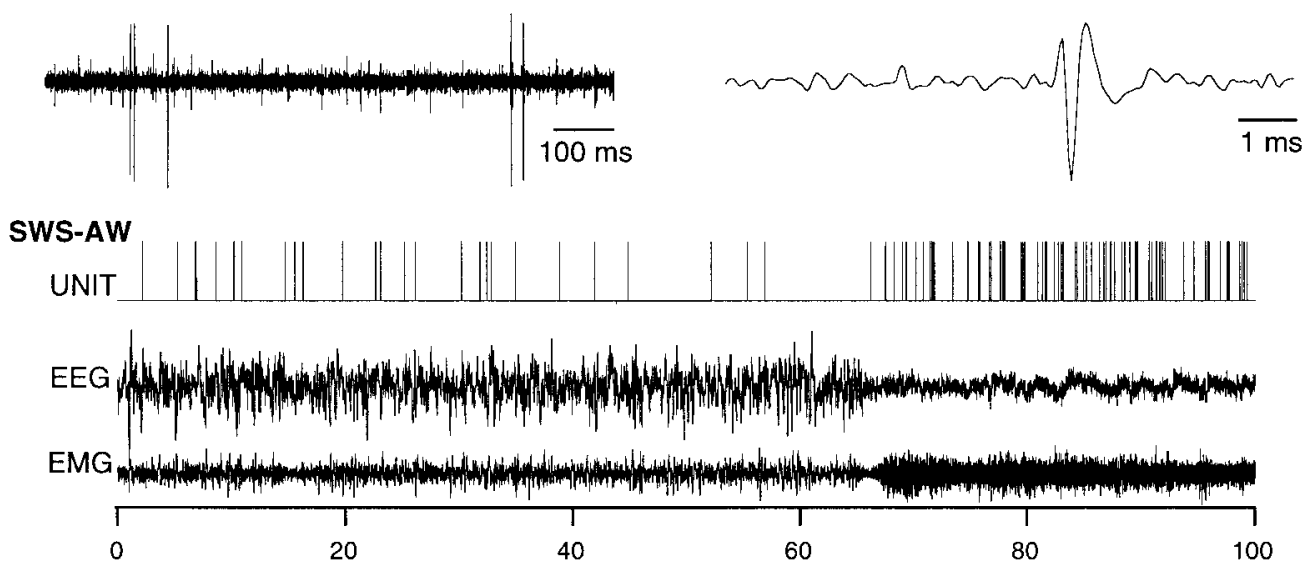

REM

UNIT IIII

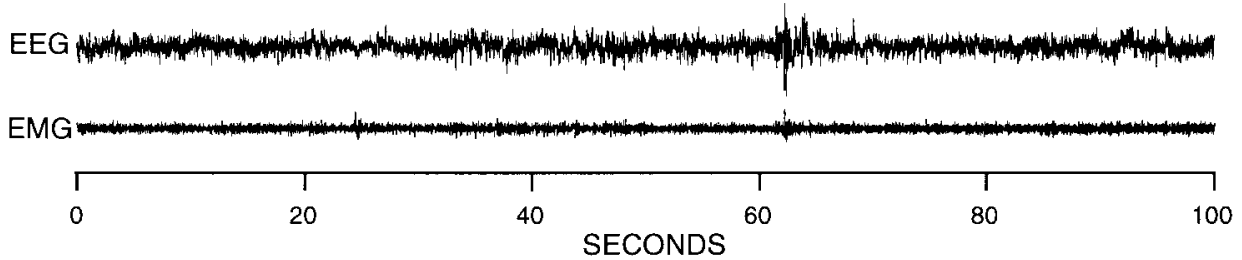

Figure 3. Simultaneous recording of VTA GABA single-unit activity, cortical EEG, and neck EMG activity. Inset on top left shows a $1 \mathrm{sec}$ filtered recording of a relatively slow VTA GABA spike, and inset on top right demonstrates an expanded time-base view of one of the filtered spike waveforms at left. A discriminated signal corresponding to each VTA GABA unit discharge (UNIT) is shown above the simultaneously recorded EEG and EMG stripcharts below. This slow VTA GABA neuron was chosen to facilitate comparisons between UNIT, EEG, and EMG. A more typical VTA GABA neuron would fill in the UNIT space with activity, regardless of state. The top stripchart recording shows the activity of the VTA GABA neuron during SWS to AW, and the bottom strip-chart recording shows its activity during REM. Note that during SWS the EEG is of relatively large amplitude and slow relative to AW and REM. During AW the EMG is of relatively large amplitude compared with REM, which is characterized by neck muscle atonia. The UNIT activity corresponding to the VTA GABA neuron spike is low during SWS and high during AW and REM.

\section{Correlations between VTA GABA neuronal activity and electrocortical activity}

The decrease in VTA GABA neuron firing during SWS and the increase during REM relative to AW was not accompanied by synchronized rhythmic activity. In other words, they did not exhibit instantaneous or rhythmic firing (bimodal distribution of interspike intervals) at the same frequency as retrosplenial electrocortical $\delta(1-4 \mathrm{~Hz})$ activity during SWS (mean SWS firing rate $=12.9 \pm 2.6 \mathrm{~Hz} ; n=25)$ or $\alpha(8-18 \mathrm{~Hz})$ activity during AW. However, their instantaneous and average firing rate were within the broad frequency range of $\gamma$ activity $(30-58 \mathrm{~Hz})$ during REM (mean REM firing rate $=37.9 \pm 5.6 \mathrm{~Hz} ; n=25$ ). Despite the marked slowing of VTA GABA neurons during SWS, the instantaneous firing rate of VTA GABA neurons was rarely correlated with $\delta$ activity, the predominant EEG frequency of the retrosplenial cortex. With the possible exception of the correlation between instantaneous firing rate and $\gamma$ activity during REM, VTA GABA neuron unit activity, as determined by inspection of the first-order interval spike histograms or autocorrelograms, showed no rhythmic activity in association with $\alpha$ activity during AW or $\delta$ activity during SWS. Nonetheless, to more closely examine the possibility that the unit discharge activity might be correlated with electrocortical activity, we performed STA of VTA GABA neuron discharges during AW, SWS, and REM sleep. As shown in Figure 6, there appeared to be little correlation between unit firing and retrosplenial EEG activity.

\section{Correlations between VTA GABA neuronal activity and electrocortical activity after sleep deprivation}

Sleep deprivation produces an increase in $\delta$ wave power during deprived sleep relative to normal sleep (Rosenberg et al., 1976; Borbely et al., 1981; Lancel et al., 1991). Because VTA GABA neuron firing rate decreased during SWS and increased during REM relative to AW, we sought to determine whether VTA GABA neuron activity correlated with the changes in EEG power produced by sleep deprivation. Figure 7 shows the simultaneous $\delta$ and $\gamma$ band activity associated with the firing rate of two VTA GABA neurons recorded from two separate sleep-deprived rats. There is a marked increase in $\delta$ activity during SWS and a mild increase in $\gamma$ activity during REM, but not AW. As during normal sleep, deprived sleep VTA GABA neuron firing appears to be activity dependent during AW, low during SWS, and enhanced during REM sleep. Figure 8 summarizes the effects of deprived sleep on EEG band power and VTA GABA neuron firing rate, as well as the correlation between the changes that occurred in EEG band power versus the changes that occurred in VTA GABA neuron firing rate during AW, SWS, and REM. Compared with the last episode of sleep before deprivation (Fig. $8 A$ ), deprived-sleep $\alpha$ activity during AW increased significantly $\left(p=0.013 ; F_{(2,11)}=14.183\right.$; mean normal sleep $8-18 \mathrm{~Hz}$ power $\left.=0.22 \pm 0.02 \mathrm{mV}^{2} / \mathrm{Hz}\right), \delta$ activity during SWS increased significantly $\left(p=0.004 ; F_{(2,11)}=35.807\right.$; mean normal sleep 1-4 $\mathrm{Hz}$ power $\left.=3.9 \pm 0.33 \mathrm{mV}^{2} / \mathrm{Hz}\right)$, and $\gamma$ activity during REM was not significantly affected $\left(p=0.253 ; F_{(2,11)}=1.664\right.$; mean REM sleep $30-58 \mathrm{~Hz}$ power $=0.07 \pm 0.009 \mathrm{mV}^{2} / \mathrm{Hz}$ ). The firing rate of VTA GABA neurons was averaged during the same epochs corresponding to the EEG analysis above. Compared with the last episode of sleep before deprivation, deprived-sleep VTA GABA neuron firing rate (Fig. $8 B$ ) was not significantly ( $p=$ 0.956; $F_{(2,33)}=0.003$ ) affected during AW (mean normal sleep AW firing rate $=34.2 \pm 7.8 \mathrm{~Hz})$, decreased significantly $(p=$ $0.011 ; F_{(2,33)}=8.277$ ) during SWS (mean normal sleep SWS firing rate $=16.1 \pm 3.4 \mathrm{~Hz}$ ), but was not significantly $(p=0.102$; $F_{(2,33)}=3.009$ ) affected during REM (mean normal sleep REM firing rate $=43.4 \pm 7.3 \mathrm{~Hz}$ ). Figure $8 C$ summarizes the relationship between the change in VTA GABA neuron firing rate and the change in $\alpha, \delta$, and $\gamma$ power corresponding to deprived versus normal AW $\alpha$ activity, SWS $\delta$ activity, and REM $\gamma$ activity, respectively. Each point represents the average change in firing 
Figure 4. VTA GABA neuron spontaneous firing rate during multiple sleep-wake cycles. Inset on top left shows a $1 \mathrm{sec}$ filtered recording of a typical VTA GABA spike, and inset on top right demonstrates an expanded time-base view of one of the filtered spike waveforms. The four strip-charts show a continuous rate meter record demonstrating the firing rate of this VTA GABA neuron over $3 \mathrm{hr}$ during multiple sleep-wake cycles. Each vertical bar represents the firing rate over $10 \mathrm{sec}$. The horizontal bars indicate the state of sleep as determined from the simultaneously recorded EEG, EMG, and behavioral responses. During AW/QW (small horizontal line) the firing pattern is irregular and marked by paroxysms of increased firing rate associated with the subject's movement in the open-field chamber. During SWS (small dashed horizontal line) the firing rate is consistently regular and relatively slow. During most of REM episodes (large horizontal line) the firing rate is relatively high and persistently elevated compared with AW/QW. The most dramatic increase in REM is evident in the right-most REM episode in the bottom rate meter record.
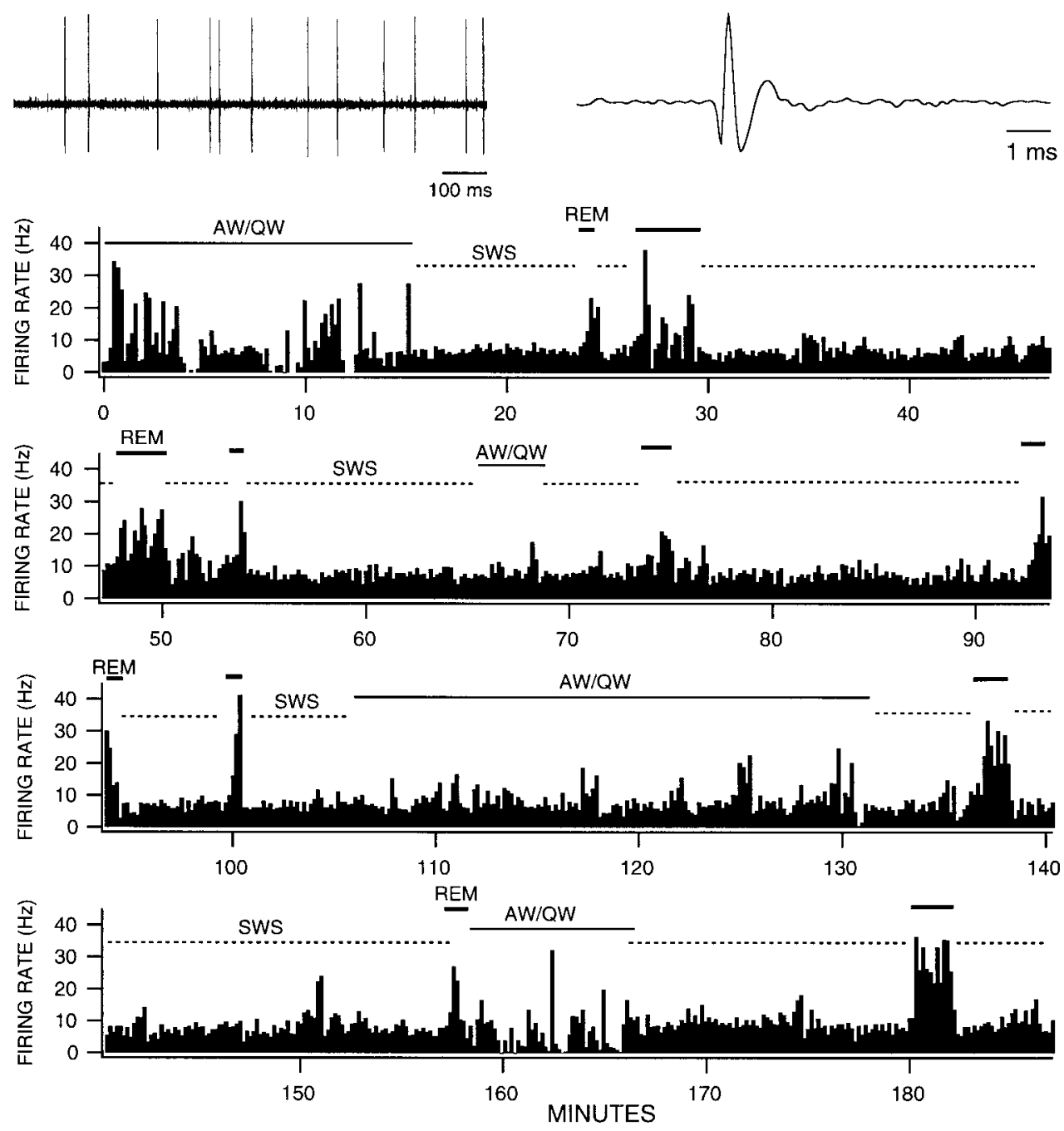

rate of all VTA GABA neurons recorded in a particular rat (one point per rat) and the average change of power for each of the bands. There was a mild correlation between the increase in $\delta$ wave power and the decrease in VTA GABA neuron firing rate during SWS $(r=0.658 ; p<0.05)$.

VTA GABA neuron firing rate was also correlated with changes in total EEG rms voltage. Compared with the last episode of sleep before deprivation, deprived-sleep EEG rms during AW increased significantly $\left(p=0.0025 ; F_{(2,11)}=31.23\right.$; mean normal AW rms $\mathrm{V}=0.34 \pm 0.02 \mathrm{mV})$, during SWS increased significantly $\left(p=0.00001 ; F_{(2,11)}=325.456\right.$; mean normal SWS rms $\mathrm{V}=0.53 \pm 0.02 \mathrm{mV})$, and during REM increased significantly $\left(p=0.004 ; F_{(2,11)}=24.270\right.$; mean normal REM rms V = $0.4 \pm 0.03 \mathrm{mV})$. Similar to $\delta$ wave activity and VTA GABA neuron slowing, there was a mild correlation between the increase in rms voltage and the decrease in VTA GABA neuron firing rate during SWS $(r=0.696 ; p<0.05)$.

\section{DISCUSSION}

The most distinguishing feature of VTA GABA neuron spontaneous activity recorded in halothane-anesthetized rats was their uninterrupted phasic activity characterized by alternating $0.5-2.0$ sec ON/OFF periods (Steffensen et al., 1998). In freely behaving rats, phasic activity was not observed, and the firing rate, on average, was greater than in halothane-anesthetized rats (i.e.,
$33 \pm 5 \mathrm{~Hz}$ vs $19 \pm 2 \mathrm{~Hz}$ ). Although not quantified in this study, the firing rate of these neurons was modulated during movement, often associated with the initiation of certain head or forelimb movements or onset of waking, but not during sustained locomotor activity, because VTA GABA neuron firing rate is not modulated during traverse of a 5 foot runway for reward (R. A. Gallegos, S. C. Steffensen, J. R. Criado, R.-S. Lee, and S. J. Henriksen, unpublished observation). We have observed VTA GABA neuron spontaneous firing rates exceeding $100 \mathrm{~Hz}$ during specific motor behaviors or during REM sleep, a rate that is consistent with their short refractory period and lack of spike accommodation (Steffensen et al., 1998).

Although general anesthetics do not significantly affect the spontaneous firing rate of midbrain DA neurons, they reduce their characteristic bursting activity and alter their sensitivity to DA receptor agonists and drugs of abuse (Bunney et al., 1973a,b; Mereu et al., 1984; Kelland et al., 1990). In contrast, the firing rate of VTA GABA neurons was reduced significantly by the three anesthetics quantified in this study and abolished by others not quantified, including the fast-acting and slow-acting barbiturates (S. C. Steffensen, R.-S. Lee, and S. J. Henriksen, unpublished observation). VTA GABA neuron firing rate was depressed most by chloral hydrate, then ketamine, and then halothane. All of these anesthetics produced adequate anesthesia, as determined by 
A

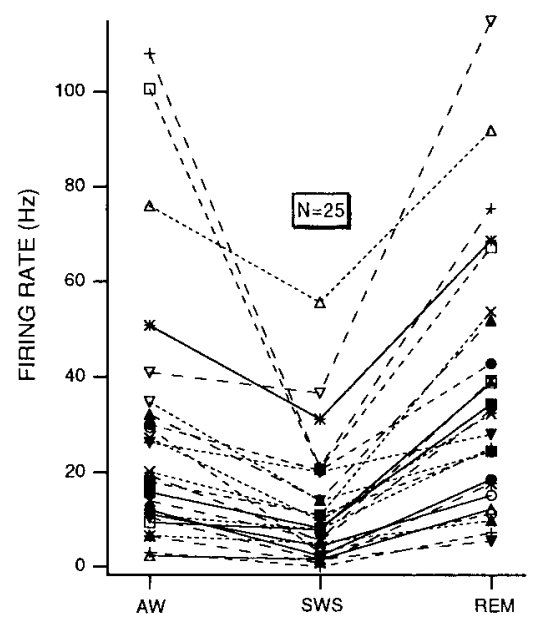

B

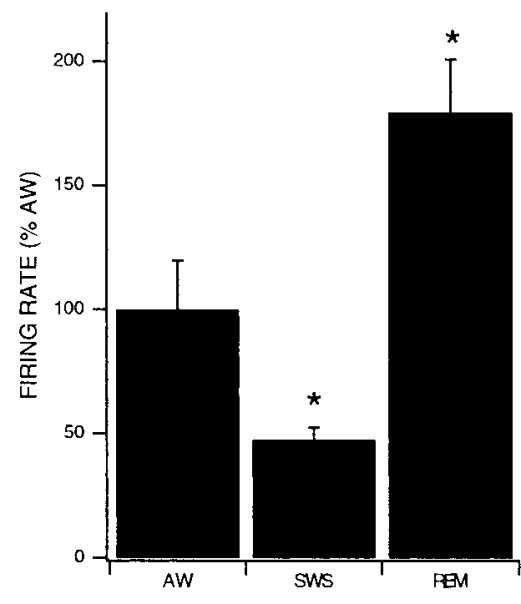

Figure 5. Summary of VTA GABA neuron spontaneous firing rate during slow-wave sleep and REM sleep compared with active wakefulness. $A$, Firing rates for VTA GABA neurons were sampled in $10 \mathrm{sec}$ epochs randomly during all states, and a minimum of 12 epochs was taken for each state for determinations of firing rate. All of the 25 VTA GABA neurons that were studied demonstrated decreased firing rates during SWS relative to AW. Most of the 25 VTA GABA neurons that were studied showed increased firing rates during REM relative to AW. $B$, This graph summarizes the firing rates of VTA GABA neurons during SWS and REM compared with AW. VTA GABA neuron mean firing rate was significantly decreased during SWS and significantly increased during REM sleep, relative to AW. Asterisks indicate significance level $p<0.05$. the lack of reflex response to tail pinch. Adequate anesthesia not only depressed VTA GABA neuron firing rate but induced pronounced phasic ON/OFF activity similar to that reported previously in rats maintained on halothane (Steffensen et al., 1998). These results demonstrate that VTA GABA neurons are especially sensitive to anesthetics and that anesthetics induce a pattern of discharge activity that differs significantly from that during SWS, wherein the discharge activity of VTA GABA neurons was also slow, but regular, and nonphasic.

The activity of VTA GABA neurons was studied during the normal sleep-wake cycle to evaluate their relationship to cortical arousal. Relative to AW, VTA GABA neuron firing rate decreased 53\% during SWS. Although VTA GABA neuron unit discharge slowed during both SWS and anesthesia, we could not distinguish whether the decreased rate resulted from reduced afferent input or from intrinsic decreases in the excitability of VTA GABA neurons. However, because we have demonstrated previously that the firing rate of VTA GABA neurons is highly dependent on excitatory synaptic input from NMDA receptormediated excitatory afferents (Steffensen et al., 1998), it is likely that the slowing results, at least in part, from diminished glutamatergic input. VTA GABA neuron unit discharge increased $79 \%$ during REM sleep, a state characterized by an inhibition of EMG activity and decreased responsiveness to external stimuli (Wu et al., 1989). This observation indicates that it is possible for the discharge of VTA GABA neurons to increase independently of motor activity or sensory input. Furthermore, changes in gross locomotor activity exhibited little correlation with VTA GABA neuron firing rate, providing further evidence that their activity does not merely reflect changes in motor output.

Although the rate or pattern of firing of midbrain DA neurons appears to be unaltered during the sleep-wake cycle (Miller et al., 1983; Steinfels et al., 1983), it has been demonstrated that non-DA neurons in the substantia nigra reticulata $(\mathrm{SNr})$ and VTA evince increased firing rates during REM compared with SWS and in AW compared with QW (Miller et al., 1983). However, there was no significant difference in the firing rate of VTA or SNr non-DA neurons during REM sleep stage compared with AW (Miller et al., 1983). In contrast, here we report a significant increase in the firing rate of VTA GABA neurons during REM sleep compared with AW. The activity of this homogeneous population of VTA GABA neurons is modulated differentially during the sleep-wake cycle and preferentially during REM

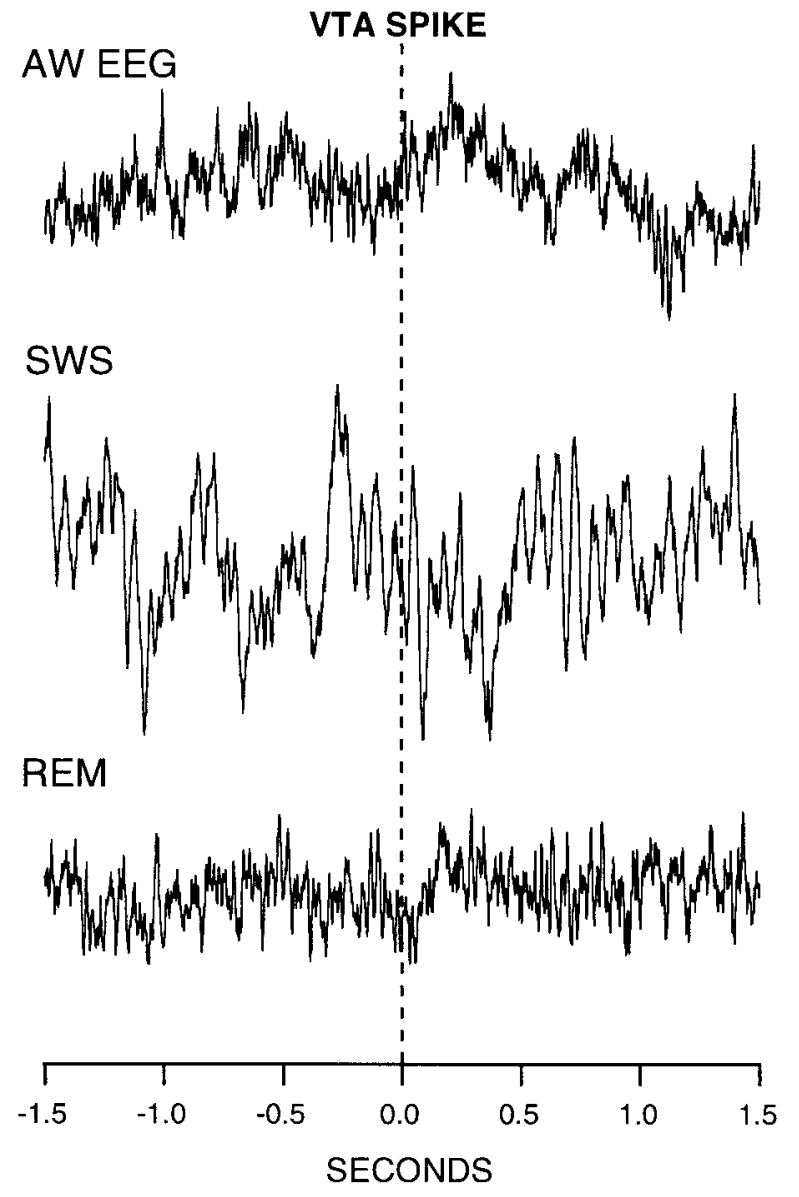

Figure 6. Lack of correlation between VTA GABA neuron spike discharge and EEG activity. The three traces show spike-triggered averaging of unit-EEG cross-correlation for a single neuronal potential recorded in a representation rat across sleep-wake states. VTA unit spikes (100 individual events) were used to average the pre-unit and post-unit discharge EEG activity during each of the three states, AW, SWS, and REM. Regardless of state, there was no correlation between unit activity and EEG activity. The dashed zero-line is the time of occurrence of VTA GABA neuron spike. 


\section{A}

1-4 Hz EEG POWER (mV^2/Hz)

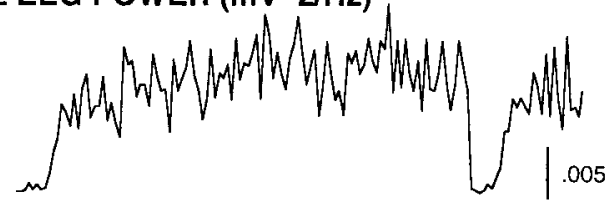

$30-58 \mathrm{~Hz}$

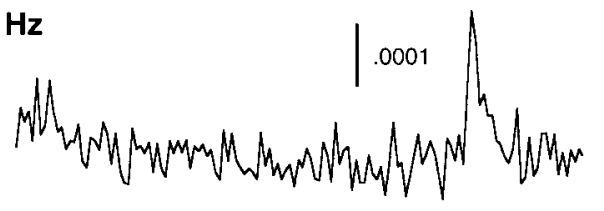

VTA GABA NEURON

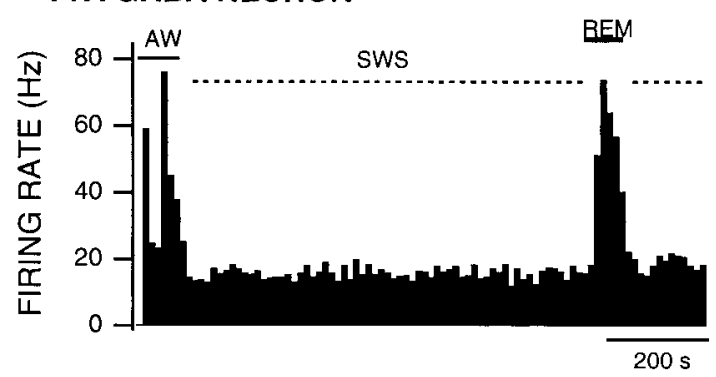

B
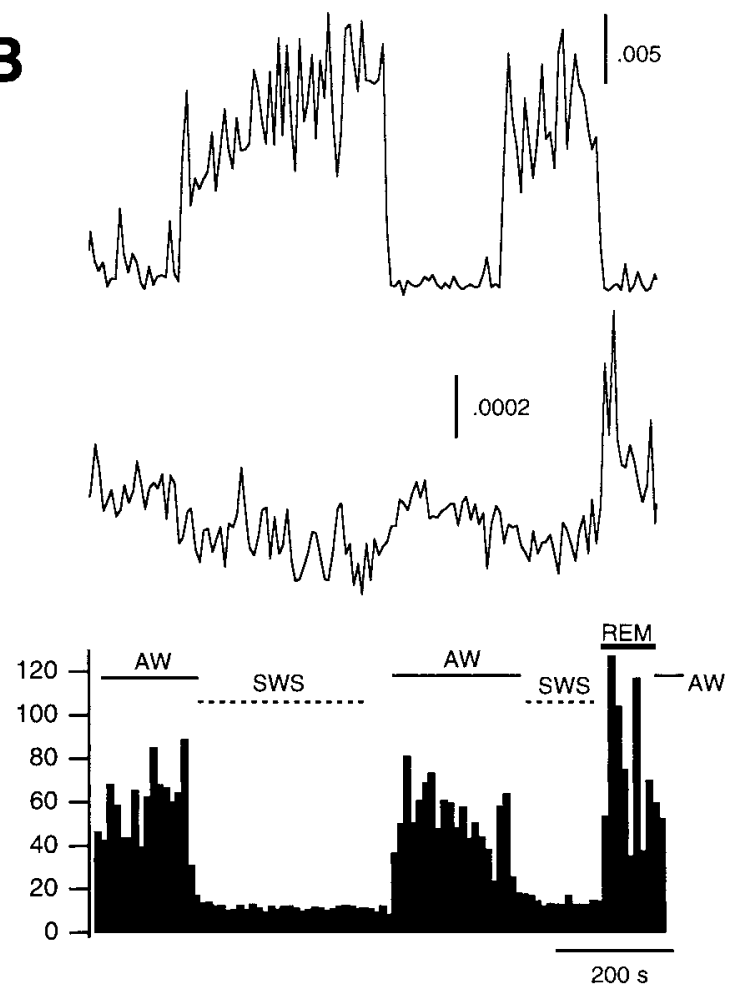

Figure 7. Cortical electroencephalographic spectral band activity and VTA GABA neuron firing rate. $A$, $B$, EEG spectral band activity and VTA GABA neuron firing rate are shown for two sleep-deprived rats. In both rats, retrosplenial $\delta$ wave activity (1-4 Hz) was greatest during SWS relative to AW and REM and increased progressively during each SWS episode. Notwithstanding the relative amplitude and signal-to-noise ratio, retrosplenial $\gamma$ wave activity $(30-58 \mathrm{~Hz})$ was of greatest amplitude during REM sleep, relative to AW and SWS. VTA GABA neuron firing rate was directly correlated with the EEG spectral band activity, being low when $\delta$ wave activity was high and high when $\gamma$ activity was high. Note that there is a small yet progressive slowing of VTA GABA neuron activity during the progressive increase in $\delta$ wave activity (seen best during the first episode of SWS in $B$ ). Temporal correlations can be drawn between EEG spectral band activity, designation of sleep state, and VTA GABA neuron activity. EEG spectral band power was determined at $4.0 \mathrm{sec}$ epochs.

sleep, when motor responses are "paralyzed," suggesting that they do not subserve motor behaviors per se but are involved in psychomotor-related events underlying cortical arousal.

We explored a possible causal relationship between VTA GABA neuron firing and cortical activation by correlating unit activity with EEG spectral band activity during AW, SWS, and REM sleep. VTA GABA neuron spiking was not rhythmically synchronized with $\alpha, \delta$, or $\gamma$ activity during AW, SWS, or REM, respectively. However, the instantaneous and average firing rates of VTA GABA neurons were correlated temporally with $\gamma$ activity during REM, indicating a link between unit activity and retrosplenial EEG activity. Whether VTA GABA neuron activity contributed to or just reflected the cortical rhythm was beyond the scope of this study. Such determinations likely require in situ pharmacological or experimental manipulations of neuronal activity.

$\delta(1-4 \mathrm{~Hz})$ wave EEG activity is a function of previous waking. During SWS, $\delta$ activity is maximal at the beginning of the sleep period and declines progressively during the sleep period (Rosenberg et al., 1976; Borbely et al., 1981; Lancel et al., 1991). After sleep deprivation, $\delta$ activity is enhanced, especially in the first part of deprived sleep (Rosenberg et al., 1976; Borbely et al., 1981; Tobler and Borbely, 1986; Lancel et al., 1991). Indeed, in humans and rats, the rate of rise and peak response of $\delta$ activity during SWS increases after sleep deprivation (Trachsel et al., 1989; Dijk et al., 1990). We found that $\delta$ activity during SWS increased nearly threefold in deprived sleep versus normal sleep.
Concomitant with the increase in $\delta$ activity was a decrease in VTA GABA neuron firing rate. In fact, there was a mild correlation between the degree of increase in $\delta$ activity and the degree of slowing of VTA GABA neuron activity, suggesting a link between VTA GABA neuron activity and cortical arousal (Fig. 8). Enhanced $\theta$ wave power has also been observed during REM sleep in deprived rats (Borbely et al., 1984; Tobler and Borbely, 1986). It was hypothesized that, similar to the regulation of SWS, REM recovery results from an increase in both duration and intensity of $\theta$; however, more recent studies have failed to find consistent elevations in $\theta$ activity during REM recovery (Lancel et al., 1992). $\gamma$ wave $(30-58 \mathrm{~Hz})$ activity and $\theta$ activity covary across the sleep-wake cycle, being high during AW and REM and low during SWS or QW (Maloney et al., 1997). It also reflects cortical arousal, independent of motor activity, attaining maximal levels during REM, when EMG activity is minimal. It was proposed that the covariation of $\gamma$ and $\theta$ activity across states and behaviors suggests that a common system may modulate these fast and slow EEG rhythms and that such modulation, potentially emanating from the basal forebrain (Maloney et al., 1997), could predominate during certain states or behaviors, such as during REM sleep. We did not observe a significant correlation between the degree of increase of $\gamma$ activity and the degree of increase of VTA GABA neuron firing rate after sleep deprivation, likely because of the lack of significant change in $\gamma$ activity during deprived REM.

Early stimulation (Moruzzi and Magoun, 1949) and lesion 


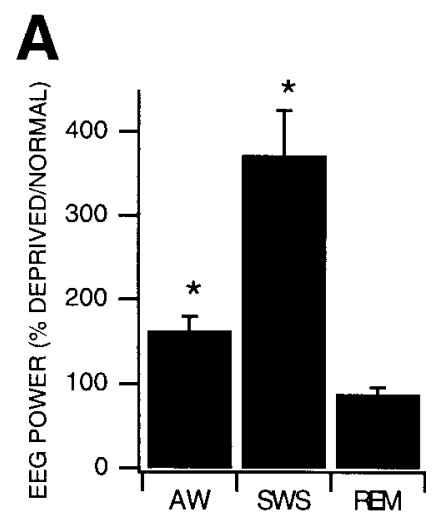

$(\alpha)$

$(\delta)$

$(\gamma)$

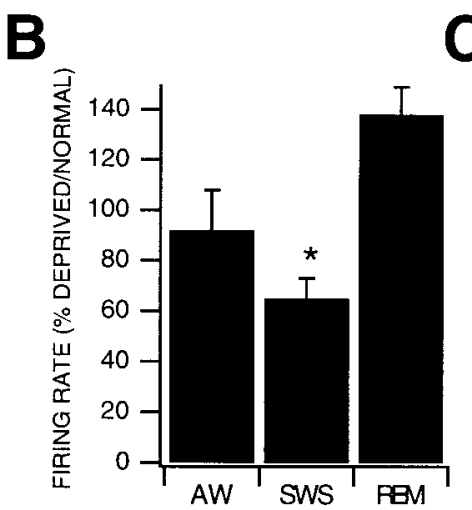

$(\alpha) \quad(\delta)$

$(\gamma)$

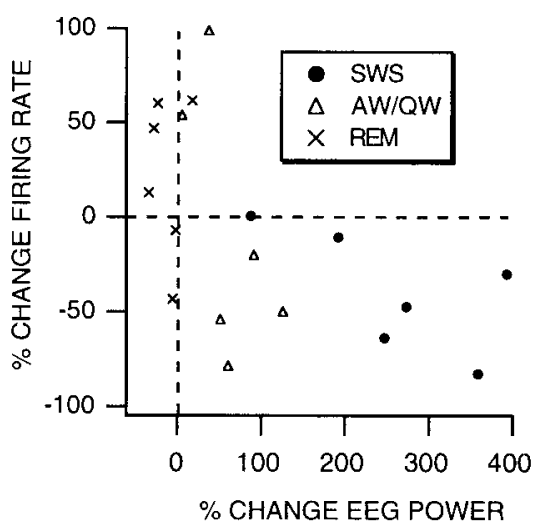

Figure 8. Relationship between electroencephalographic spectral power and VTA GABA neuron firing rate: deprived sleep versus normal sleep. A, This graph summarizes the effects of $24 \mathrm{hr}$ sleep deprivation on EEG spectral power during the sleep-wake cycle. Although $\alpha$ wave activity ( $\alpha$ : $8-18$ $\mathrm{Hz})$ during $\mathrm{AW}$ and $\delta(\delta: 1-4 \mathrm{~Hz})$ wave activity during SWS were significantly increased during deprived sleep relative to normal sleep, $\gamma(\gamma: 30-58 \mathrm{~Hz})$ wave activity during REM sleep was not significantly altered (expressed as percentage deprived vs normal sleep). Asterisks equal significance level $p<$ 0.05. B, This graph summarizes the effects of $24 \mathrm{hr}$ sleep deprivation on VTA GABA neuron activity during the sleep-wake cycle. Although there was no significant difference in VTA GABA neuron firing rate during deprived AW $\alpha$ wave activity in deprived rats, there was a significant slowing during deprived SWS $\delta$ wave activity relative to normal sleep. Asterisk equals significance level $p<0.05$. C, This graph plots the change in VTA GABA neuron firing rate on the abscissa versus the change in EEG spectral band power for each state of the sleep-wake cycle in deprived sleep versus normal sleep. Note that VTA GABA neuron slowing increases as a function of increased $\delta$ wave activity during SWS. There is no clear relationship between the change in VTA GABA neuron firing and the change in $\alpha$ or $\gamma$ activity during either AW or REM sleep, respectively. Each point within each category represents a different rat.

(Bach-Y-Rita et al., 1966) studies have implicated the midbrain reticular formation, including the VTA, in the electrocortical and behavioral activation that characterize wakefulness. However, studies involving more selective lesions of the reticular formation have revealed a dissociation between behavioral and electrocortical activation (Feldman and Waller, 1962; Jones et al., 1973), indicating that distinct subareas of the rostral brainstem core underlie their respective mechanisms. Cholinergic neurons in the basal forebrain serve as the extrathalamic relay from the reticular formation to the cerebral cortex and have been shown to be critically involved in the regulation of cortical activity and behavioral state (Krnjevic and Phillis, 1963; Jones, 1993). Recently, it has been demonstrated that corticopetal cholinergic and GABAergic neurons in the basal forebrain fire rhythmically or are correlated with cortical EEG activity (Duque et al., 2000; Manns et al., 2000). Although it remains to be definitively established whether basal forebrain neuronal activity is contributory to or reflective of cortical activity, these findings provide strong evidence for a role for basal forebrain neurons in regulating extrathalamic cortical activation.

Although VTA GABA neuron firing was directly correlated with the sleep-wake cycle, there was no evidence of specific activity preceding or lagging each state or of synchronous activity in association with the cortical EEG. The mere concurrence of VTA GABA neuronal activity with cortical activation is not enough to establish causal or mechanistic connections between neuronal activity and electrocortical or behavioral activation. However, VTA GABA neurons may still be important regulators or switches of extrathalamic electrocortical or behavioral activation. VTA GABA neurons, including their projections and their inputs, similar to the role of $\mathrm{SNr}$ or $\mathrm{SNc}$ GABA neurons in regulating motor output, are in a critical position to modulate DA psychomotor output as integrators of convergent information from sensory, cortical, and limbic areas. The tonic glutamatergic input that regulates the firing of VTA GABA neurons may function in a manner similar to the role played by subthalamic inputs to SNr GABAergic neurons in mediating SNr inhibition of SNc DA neurons (Tepper et al., 1995). Alternatively, by virtue of their widespread axonal distribution and their wide dynamic range, VTA GABA neurons may be involved, independent of DA neurons, in the reticular activating system for extrathalamic regulation of cortical activity.

\section{REFERENCES}

Bach-Y-Rita G, Baurand C, Christolomme AA (1966) A comparison of EEG modifications induced by coagulation of subthalamus, preoptic region and mesencephalic reticular formation. Electroencephalogr Clin Neurophysiol 26:493-502.

Borbely AA, Baumann F, Brandeis D, Strauch I, Lehmann D (1981) Sleep deprivation: effect on sleep stages and EEG power density in man. Electroencephalogr Clin Neurophysiol 51:483-495.

Borbely AA, Tobler I, Hanagasioglu M (1984) Effect of sleep deprivation on sleep and EEG power spectra in the rat. Behav Brain Res 14:171-182.

Bunney BS, Aghajanian GK, Roth RH (1973a) Comparison of effects of 1-dopa, amphetamine and apomorphine on firing rate of rat dopaminergic neurons. Nat New Biol 245:123-125.

Bunney BS, Walters JR, Roth RH, Aghajanian GK (1973b) Dopaminergic neurons: effect of antipsychotic drugs and amphetamine on single cell activity. J Pharmacol Exp Ther 185:560-571.

Dijk DJ, Brunner DP, Borbely AA (1990) Time course of EEG power density during long sleep in humans. Am J Physiol 258:R650-661.

Duque A, Balatoni B, Detari L, Zaborski L (2000) EEG correlation of the discharge properties of identified neurons in the basal forebrain. J Neurophysiol 84:1627-1635.

Feldman SM, Waller HJ (1962) Dissociation of electrocortical activation and behavioral arousal. Nature 196:1320-1322.

Ford B, Holmes CJ, Mainville L, Jones BE (1995) GABAergic neurons in the rat pontomesencephalic tegmentum: codistribution with cholinergic and other tegmental neurons projecting to the posterior lateral hypothalamus. J Comp Neurol 363:177-196.

Freeman AS, Bunney BS (1987) Activity of A9 and A10 dopaminergic neurons in unrestrained rats: further characterization and effects of apomorphine and cholecystokinin. Brain Res 405:46-55.

Gallegos RA, Criado JR, Lee RS, Henriksen SJ, Steffensen SC (1999) Adaptive responses of GABAergic neurons in the ventral tegmental area to chronic ethanol. J Pharmacol Exp Ther 291:1045-1053.

Jones BE (1993) The organization of central cholinergic systems and their functional importance in sleep-waking states. Cholinergic function and dysfunction. Prog Brain Res 98:61-71. 
Jones BE, Bobillier P, Pin C, Jouvet M (1973) The effect of lesions of catecholamine-containing neurons upon monoamine content of the brain and EEG and behavioral waking in the cat. Brain Res 58:157-177.

Kalivas PW, Churchill L, Klitenick MA (1993) The circuitry mediating the translation of motivational stimuli into adaptive motor responses. In: Limbic motor circuits and neuropsychiatry (Kalivas PW, Barnes CD, eds), pp 237-287. Boca Raton: CRC.

Kelland MD, Chiodo LA, Freeman AS (1990) Anesthetic influences on the basal activity and pharmacological responsiveness of nigrostriatal dopamine neurons. Synapse 6:207-209.

Krnjevic K, Phillis JW (1963) Pharmacological properties of acetylcholinesensitive cells in the cerebral cortex. J Physiol (Lond) 166:328-350.

Kropf W, Kuschinsky K (1991) Electroencephalographic correlates of the sedative effects of dopamine agonists presumably acting on autoreceptors. Neuropharmacology 30:953-960.

Kropf W, Kuschinsky K, Krieglstein J (1989) Apomorphine-induced alterations in cortical EEG activity of rats. Involvement of D-1 and D-2 dopamine receptors. Naunyn Schmiedebergs Arch Pharmacol 340:718-725.

Lancel M, van Riezen H, Glatt A (1991) Effects of circadian phase and duration of sleep deprivation on sleep and EEG power spectra in the cat. Brain Res 548:206-214.

Lancel M, van Riezen H, Glatt A (1992) Enhanced slow-wave activity within NREM sleep in the cortical and subcortical EEG of the cat after sleep deprivation. Sleep 15:102-118.

Lee R-S, Steffensen SC, Henriksen SJ (1997) Ventral tegmental area non-dopamine neuronal activity during cortical arousal. Soc Neurosci Abstr 23:793.

Lin JS, Sakai K, Vanni-Mercier G, Jouvet M (1989) A critical role of the posterior hypothalamus in the mechanisms of wakefulness determined by microinjection of muscimol in freely moving cats. Brain Res 479:225-240.

Maloney KJ, Cape EG, Gotman J, Jones BE (1997) High-frequency gamma electroencephalogram activity in association with sleep-wake states and spontaneous behaviors in the rat. Neuroscience 76:541-555.

Manns ID, Alonso A, Jones BA (2000) Discharge properties of juxtacellularly labeled and immunohistochemically identified cholinergic basal forebrain neurons recorded in association with the electroencephalogram in anesthetized rats. J Neurosci 20:1505-1518.

Mereu G, Fanni B, Gessa GL (1984) General anesthetics prevent dopaminergic neuron stimulation by neuroleptics. In: Catecholamines, Part B: Neuropharmacology and central nervous system-theoretical aspects (Usdin E, Carlsson A, Dahlstrom A, Engel J, eds), pp 353-358. New York: Alan R. Liss.

Miller JD, Farber J, Gatz P, Roffwarg H, German DC (1983) Activity of mesencephalic dopamine and non-dopamine neurons across stages of sleep and waking in the rat. Brain Res 273:133-141.

Mogenson GJ (1987) Limbic-motor integration. In: Progress in psychobiology and physiological psychology, Vol 12 (Epstein A, Morrison A, eds), pp 117-170. New York: Academic.

Moruzzi G, Magoun HW (1949) Brainstem reticular formation and activation of the EEG. Electroencephalogr Clin Neurophysiol 1:455-473.

Mugnaini E, Oertel W (1985) An atlas of the distribution of GABAergic neurons and terminals in the rat CNS as revealed by GAD immunohistrochemistry. In: Handbook of chemical neuroanatomy, (Bjorklund A, Hokfelt T, eds), pp 436-608. Amsterdam: Elsevier.

Nagai T, McGeer PL, McGeer EG (1983) Distribution of GABA-T intensive neurons in the rat forebrain and midbrain. J Comp Neurol 218:220-238.

Nishikawa T, Scatton B (1985) Inhibitory influence of GABA on central serotonergic transmission. Raphe nuclei as the neuroanatomical site of the GABAergic inhibition of cerebral serotonergic neurons. Brain Res 331:91-103.

Nishino S, Mignot E (1997) Pharmacological aspects of human and canine narcolepsy. Prog Neurobiol 52:27-78.

Nitz D, Siegel J (1997) GABA release in the dorsal raphe nucleus: role in the control of REM sleep. Am J Physiol 273:R451-455.

Otterson OP, Storm-Mathisen J (1984) Neurons containing or accumulating transmitter amino acids. In: Handbook of chemical neuroanatomy (Bjoklund A, Hokfelt T, Kuhar MJ, eds), pp 141-245. Amsterdam: Elsevier.

Paxinos G, Watson C (1986) The rat brain in stereotaxic coordinates. San Diego, Academic.

Radulovacki M, Woicik WJ, Fornal C (1979) Effects of bromocriptine and alpha-flupenthixol on sleep in REM sleep deprived rats. Life Sci 24:1705-1712.

Rosenberg RS, Bergmann BM, Rechtschaffen A (1976) Variations in slow wave activity during sleep in the rat. Physiol Behav 17:931-938.

Schultz W (1986) Responses of midbrain dopamine neurons to behavioral trigger stimuli in the monkey. J Neurophysiol 56:1439-1461.

Schultz W, Apicella P, Ljungberg T (1993) Responses of monkey dopamine neurons to reward and conditioned stimuli during successive steps of learning a delayed response task. J Neurosci 13:900-913.

Sebban C, Tesolin-Decros B, Millan MJ, Spedding M (1999a) Contrasting EEG profiles elicited by antipsychotic agents in the prefrontal cortex of the conscious rat: antagonism of the effects of clozapine and modafinil. Br J Pharmacol 128:1055-1063.

Sebban C, Zhang XQ, Tesolin-Decros B, Millan MJ, Spedding M (1999b) Changes in EEG power in the prefrontal cortex of conscious rats elicited by drugs interacting with dopaminergic and noradrenergic transmission. Br J Pharmacol 128:1045-1054.

Steffensen SC, Raymond SR, Lee R-S, Henriksen SJ (1996) Ventral tegmental area neuronal activity and responsiveness to anesthesia. Soc Neurosci Abstr 22:1137.

Steffensen SC, Svingos AL, Pickel VM, Henriksen SJ (1997) Electrophysiological, neurochemical and ultrastructural characterization of ventral tegmental area GABAergic neurons. Soc Neurosci Abstr 23:115.

Steffensen SC, Svingos AL, Pickel VM, Henriksen SJ (1998) Electrophysiological characterization of GABAergic neurons in the ventral tegmental area. J Neurosci 18:8803-8815.

Steinfels GF, Heym J, Strecker RE, Jacobs BL (1983) Behavioral correlates of dopaminergic unit activity in freely moving cats. Brain Res 258:217-228

Szymusiak R, McGinty D (1986) Sleep-related neuronal discharge in the basal forebrain of cats. Brain Res 370:82-92.

Tepper JM, Martin LP, Anderson DR (1995) GABAa receptormediated inhibition of rat substantia nigra dopaminergic neurons by pars reticulata projection neurons. J Neurosci 15:3092-3103.

Tobler I, Borbely AA (1986) Sleep EEG in the rat as a function of prior waking. Electroencephalogr Clin Neurophysiol 64:74-76.

Trachsel L, Tobler I, Borbely AA (1989) Effect of sleep deprivation on EEG slow wave activity within non-REM sleep episodes in the rat. Electroencephalogr Clin Neurophysiol 73:167-171.

Trulson ME, Preussler DW (1984) Dopamine-containing ventral tegmental neurons in freely moving cats: activity during the sleep-waking cycle and effects of stress. Exp Neurol 83:367-377.

Wise RA, Rompre P-P (1989) Brain dopamine and reward. Annu Rev Psychol 40:191-225.

Wu MF, Mallick BN, Siegel JM (1989) Lateral geniculate spikes, muscle atonia and startle response elicited by auditory stimuli as a function of stimulus parameters and arousal state. Brain Res 499:7-17. 